\title{
First results from VLTI near-infrared interferometry on high-mass young stellar objects
}

\author{
Stefan Kraus ${ }^{a}$, Karl-Heinz Hofmann ${ }^{b}$, Karl M. Menten ${ }^{b}$, Dieter Schertl ${ }^{b}$, Gerd Weigelt ${ }^{b}$, \\ Friedrich $\mathrm{Wyrowski}^{b}$, Anthony Meilland ${ }^{b, c}$, Karine Perraut ${ }^{d}$, Romain Petrov ${ }^{c}$, \\ Sylvie Robbe-Dubois ${ }^{c}$, Peter Schilke ${ }^{e}$ and Leonardo Testi ${ }^{f}$ \\ ${ }^{a}$ Department of Astronomy, University of Michigan, 500 Church St., Ann Arbor, MI 48103, \\ USA; ${ }^{b}$ Max-Planck-Institut für Radioastronomie, Auf dem Hügel 69, 53123 Bonn, Germany; \\ ${ }^{c}$ Laboratoire Hippolyte Fizeau, UMR 6525 Université de Nice \\ Sophia-Antipolis/CNRS/Observatoire de la Côte d'Azur, Parc Valrose, 06108 Nice Cedex 2, \\ France; ${ }^{d}$ Laboratoire d'Astrophysique de Grenoble, UMR 5571 Université Joseph \\ Fourier/CNRS, BP 53, 38041 Grenoble Cedex 9, France; \\ ${ }^{e}$ I. Physikalisches Institut, Universität zu Köln, Zülpicher Strasse 77, 50937 Cologne, Germany; \\ fINAF-Osservatorio Astrofisico di Arcetri, Largo Fermi 5, 50125 Firenze, Italy
}

\begin{abstract}
Due to the recent dramatic technological advances, infrared interferometry can now be applied to new classes of objects, resulting in exciting new science prospects, for instance, in the area of high-mass star formation. Although extensively studied at various wavelengths, the process through which massive stars form is still only poorly understood. For instance, it has been proposed that massive stars might form like low-mass stars by mass accretion through a circumstellar disk/envelope, or otherwise by coalescence in a dense stellar cluster. Therefore, clear observational evidence, such as the detection of disks around high-mass young stellar objects (YSOs), is urgently needed in order to unambiguously identify the formation mode of the most massive stars.

After discussing the technological challenges which result from the special properties of these objects, we present first near-infrared interferometric observations, which we obtained on the massive YSO IRAS 13481-6124 using VLTI/AMBER infrared long-baseline interferometry and NTT speckle interferometry. From our extensive data set, we reconstruct a model-independent aperture synthesis image which shows an elongated structure with a size of $\sim 13 \times 19 \mathrm{AU}$, consistent with a disk seen under an inclination of $\sim 45^{\circ}$. The measured wavelengthdependent visibilities and closure phases allow us to derive the radial disk temperature gradient and to detect a dust-free region inside of 9.5 AU from the star, revealing qualitative and quantitative similarities with the disks observed in low-mass star formation. In complementary mid-infrared Spitzer and sub-millimeter APEX imaging observations we detect two bow shocks and a molecular outflow, which are oriented perpendicular to the disk plane and indicate the presence of a bipolar outflow emanating from the inner regions of the system.
\end{abstract}

Keywords: techniques: interferometric - stars: pre-main-sequence - stars: individual: IRAS 13481-6124 accretion, accretion disks

\section{INTRODUCTION}

In recent years, the field of infrared interferometry has experienced dramatic technological progress, providing substantial improvements, for instance, in terms of the available baseline lengths, spectral resolution, and spectral coverage. Besides quantitative improvements, the instrumentational and infrastructure advances have also considerably extended the accessible range of astrophysical targets, enabling infrared interferometry to address key scientific areas such as high-mass star formation.

Further author information: (Send correspondence to S.K.)

E-mail: stefankr@umich.edu, Telephone: 17346157374 
Although scarce in number, massive stars $\left(>10 \mathrm{M}_{\odot}\right)$ are of fundamental importance for astrophysics. Already shortly after their birth, they start to disperse their natal molecular clouds with strong stellar outflows and photoevaporate the protoplanetary disks around nearby low-mass stars. After a short hydrogen-burning phase, the most massive stars end their evolution as supernovae, enriching the interstellar medium with heavy elements and triggering the next generation of star formation through supernova shock waves. In spite of their significance, it is still poorly understood how high-mass stars form.

Early spherically symmetric calculations suggested that the accretion scenario, which appears well established for stars with masses below $10 \mathrm{M}_{\odot}$, might not work for massive protostars due to the strong radiation pressure ${ }^{1 / 2}$. Therefore, it was proposed that high-mass stars might not form by accretion, but instead by stellar merging ${ }^{3}$. Although recent theoretical work showed that the radiation pressure limit might be overcome when considering more complex infall geometries than spherically symmetric ones ${ }^{4}$, clear observational evidence, such as the detection of compact dusty disks around massive young stellar objects, is needed to unambiguously identify the formation mode of the most massive stars.

Interferometric observations at radio wavelengths already provided fascinating insight into the distribution and kinematics of the circumstellar material, although the existing observations are still strongly limited by the achievable angular resolution of thousands to hundreds of astronomical units (AUs). For instance, millimeter observations ${ }^{6}$ with an angular resolution of $\sim 0.8^{\prime \prime}$ revealed a large $\left(\sim 10^{4} \mathrm{AU}\right)$ rotating toroidal structure and gas infall motion around the YSO G24.78+0.08. Recently, mid-infrared long-baseline interferometric observation $\$ 7 / 8$ probed the environment around two massive YSOs on scales of a few tens of AUs and could resolve the inner regions of an envelope around these stars. In order to separate the contributions from a circumstellar disk and to avoid confusion with the surrounding rotating circumstellar envelope, observations in the near-infrared around $\sim 2 \mu \mathrm{m}$ are very promising, since this wavelength regime is sensitive to the thermal emission of hot $(>1000 \mathrm{~K})$ gas and dust, probing temperatures where dust is expected to sublimate $(T \sim 1500 \ldots 2000 \mathrm{~K})$ and to form a dust-free inner hole. By forming telescope baselines up to hundreds of meters, infrared interferometric imaging can now provide the milli-arcsecond (mas) angular resolution that is required to directly resolve these inner disk regions. Compared to earlier studies on high-mass YSOs, which used conventional imaging techniques at $10 \mathrm{~m}$ class telescopes equipped with adaptive optics systems, the gain in resolving power is at least one order of magnitude, while the gain compared to state-of-the-art (sub-)millimeter disk studies is about two orders of magnitudes.

However, the technological challenges for infrared interferometry on young high-mass stars are high. First, massive YSOs are still deeply embedded in their natal clouds, which results in an extremely steep drop of the spectral energy distribution (SED) between mid-infrared and near-infrared wavelengths. Accordingly, most massive YSOs are not associated with a visual counterpart. Therefore, it is essential to use off-axis telescope guiding, as offered by the STRAP units at VLTI $1.8 \mathrm{~m}$ auxiliary telescopes (ATs), which use Avalanche Photodiodes to provide a tip-tilt correction for guide stars brighter than $V=13.5$. The VLTI $8.2 \mathrm{~m}$ unit telescopes (UTs) are equipped with MACAO adaptive optic units operating on natural guide stars down to $V=17$. These sensitivity limits and the requirement that the guide star has to be located within the isoplanatic patch $\left(\sim 1^{\prime}\right)$, makes it difficult to find suitable guide stars for many high-mass YSOs, in particular since the number of potential off-axis guide stars is also strongly reduced by the high visual extinction in high-mass star forming regions.

Recently, we have obtained first near-infrared interferometric observations of a young high-mass star ${ }^{9}$, namely IRAS 13481-6124. This star has a nearby foreground star $(V=12.6)$ at a separation of $\sim 17^{\prime \prime}$, which can be used for telescope guiding even with the ATs. Furthermore, IRAS 13481-6124 is located at a very favourable celestial location $\left(\mathrm{DEC}=-61^{\circ}\right)$ which results in rather circular $u v$-tracks, providing optimal prerequisits for earth-rotation aperture-synthesis imaging. Earlier studies have estimated that IRAS 13481-6124 is located at a distance of about $3.5 \mathrm{kpc}^{10}$ and harbours a central object with a mass of $\sim 20 \mathrm{M}_{\odot}$, which is embedded in a cloud with a total mass of $\sim 1500 \mathrm{M}_{\odot} \frac{12}{\text {. }}$ 

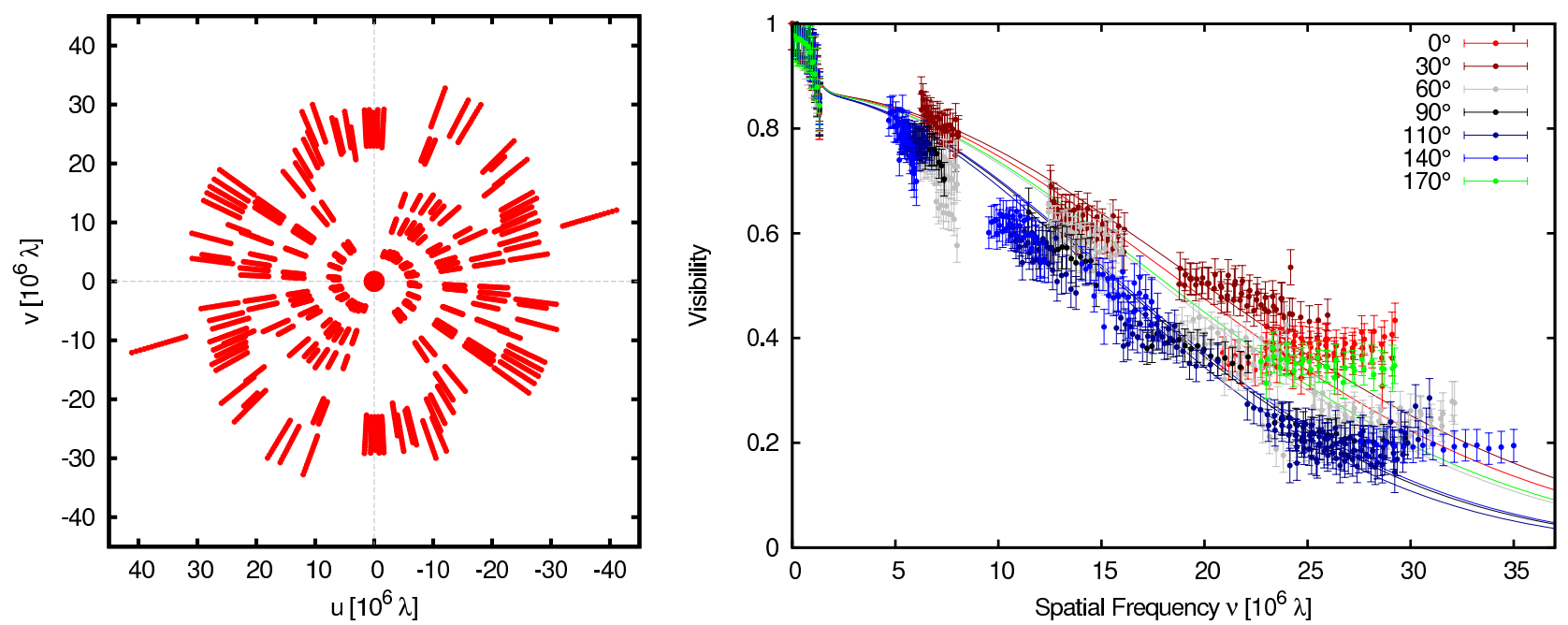

Figure 1. Left: $u v$-plane coverage obtained with our VLTI/AMBER and NTT/speckle observations. Right: Visibilities recorded with our VLTI/AMBER and NTT/speckle observations towards different position angles, revealing a strong position angle-dependence of the visibility function.

\section{OBSERVATIONS}

We observed IRAS 13481-6124 during seven nights in 2008 and 2009 using the near-infrared three-telescope beam combination instrument AMBER ${ }^{[13}$ at ESO's Very Large Telescope Interferometer (VLTI). Our spectrointerferometric observations cover the near-infrared $K$-band between $\sim 1.95$ to $2.55 \mu \mathrm{m}$ with a spectral resolution $\lambda / \Delta \lambda=35$. Using the VLTI/ATs on three different array configurations (E0-G0-H0, D0-H0-G1, A0-D0-H0) and at various hour angles, we could achieve a relatively good uv-coverage (Fig. 1, left), including baseline lengths between 12 and $85 \mathrm{~m}$. In order to cover spatially extended structures, we complemented the VLTI long-baseline interferometric observations with bispectrum speckle interferometric observations $s^{14}$ [15, yielding precise visibility information for baselines $\lesssim 3.5 \mathrm{~m}$. The speckle $K$-band data was recorded with ESO's New Technology Telescope (NTT) and our visitor camera, employing a Rockwell HAWAII detector. Following the standard observing procedure, both the VLTI and speckle observations on IRAS 13481-6124 were interlayed with calibrator observations in order to derive absolute calibrated visibilities and closure phases, which we then used for our image reconstruction and model fitting approach.

Besides these high-resolution $K$-band continuum observations, which directly trace the thermal dust emission and scattered light in the circumstellar environment around IRAS 13481-6124, we searched for signatures of mass outflow on larger spatial scales. For this, we obtained archival Spitzer/IRAC images which were recorded in the course of the GLIMPSE survey 16 and which cover wavelengths around 3.6, 4.5, 5.8, and $8.0 \mu \mathrm{m}$. In the IRAC $4.5 \mu \mathrm{m}$ image (codes as green color in the color composite shown in Fig. 2A) we detect two bow shock structures which are oriented along position angle $31 \pm 6^{\circ}$ and likely indicate $\mathrm{H}_{2}$ shock-tracer line emission ${ }^{17}$. In order to trace a potential molecular outflow, we obtained a ${ }^{12} \mathrm{CO}(3-2,346 \mathrm{GHz})$ map using the SHFI heterodyne receiver at the APEX $12 \mathrm{~m}$ telescope located on the Chajnantor plateau in the Chilean Andes. The In the obtained CO channel maps, we detect a bipolar outflow oriented along position angle $26 \pm 9^{\circ}$, with the approaching (blue-shifted) lobe southwest of IRAS 13481-6124 (Fig. 21B).

\section{APERTURE SYNTHESIS IMAGING}

Given that our observations provide a good $u v$-coverage and include closure phase information, we could use our data to reconstruct a model-independent aperture synthesis image. For this purpose, we employed the Building Block Mapping (BBM) image reconstruction algorithm $\frac{18}{18}$. This algorithm has already been employed successfully in various earlier projects to reconstruct interferometric images from simulated ${ }^{[19 \mid 20}$ and long-baseline interferometric data 21223 . The reconstruction was performed on a $256 \times 256$ grid using a pixel scale of 0.85 mas/pixel and convergence was reached after $\sim 12000$ iteration steps. In order to maximize the $u v$-coverage for imaging, we 


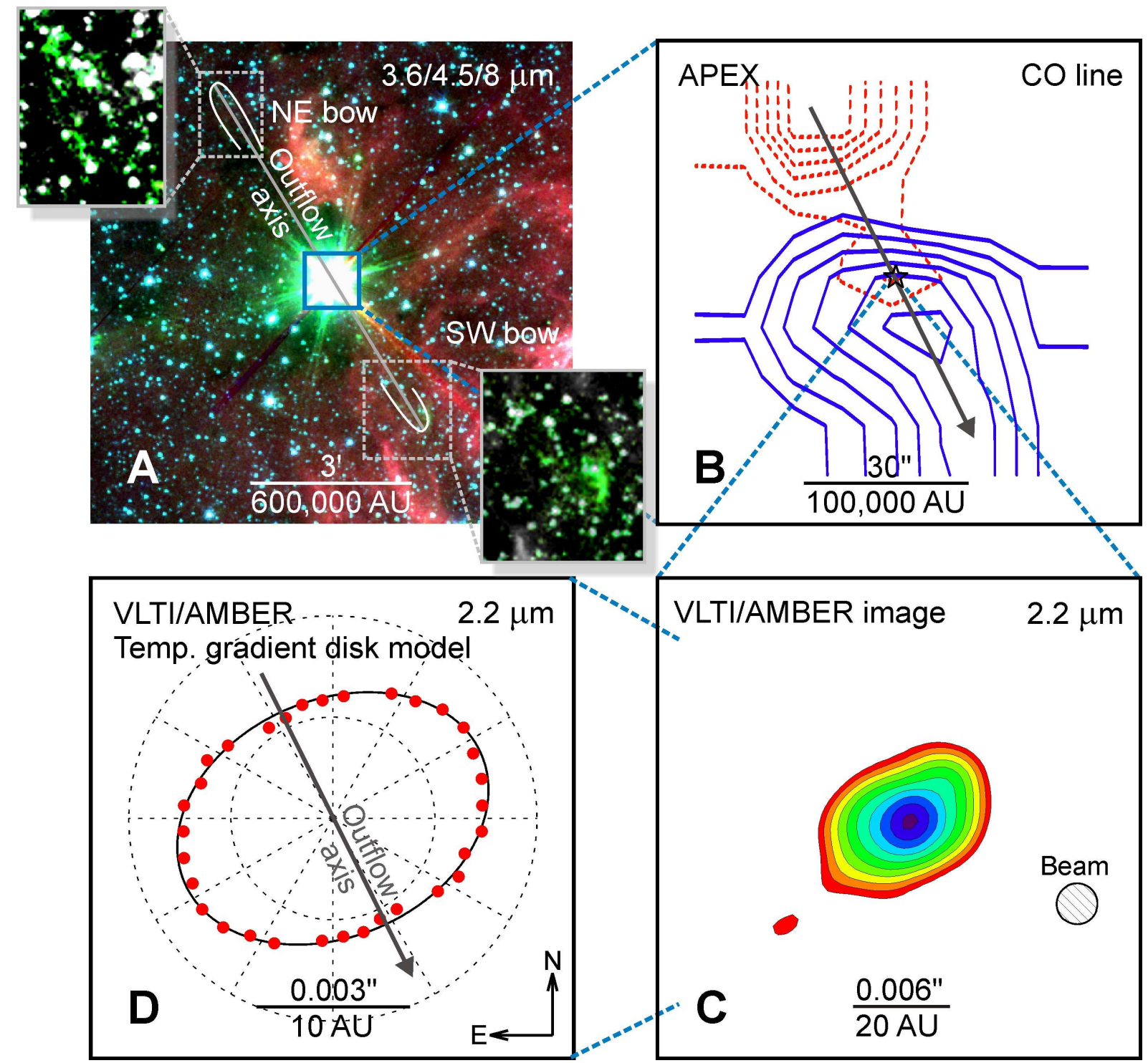

Figure 2. Zoom in on IRAS 13481-6124, covering spatial scales over more than five orders of magnitudes: (A) In Spitzer/IRAC images, we detect two bow-shock structures, indicating a collimated outflow. (B) The outflow is also detected in molecular line emission using APEX/SHFI on scales of a few $10000 \mathrm{AU}$ (the contours show blue-shifted velocities of $-10 \mathrm{~km} \mathrm{~s}^{-1}$ and redshifted velocities of $+5 \mathrm{~km} \mathrm{~s}^{-1}$, where the latter are indicated with dashed lines). (C) Applying our image reconstruction algorithm to the VLTI/AMBER data, we reconstructed a model-independent aperture synthesis image of IRAS 13481-6124. The image reveals an elongated structure, which is oriented perpendicular to the outflow direction (contours decrease from peak intensity by factors of $\sqrt{2}$ ). (D) Elongation of the compact emission component, as determined with our temperature gradient disk model. 
combined data from all spectral channels in order to reconstruct one monochromatic image. The obtained image was convolved with a Gaussian of FWHM 2.4 mas, corresponding to a resolution of $\lambda / 2 B$, where $\lambda$ denotes the observing wavelength and $B$ the length of the longest employed baseline.

The reconstructed image (Fig. 2C) has a reduced goodness-of-fit value of 1.5 and 1.0 for the squared visibilities and the closure phases, respectively. The residuals are well distributed, i.e. they closely follow a normal distribution. The image clearly reveals an elongated structure which is oriented in north-western direction. Besides this compact component, some flux appears distributed over the whole image. Measuring the flux in different apertures, we find that $80 \%$ of the total image flux is contained in a 7.0 mas aperture, while the remaining flux elements are spread rather homogeneously over the image.

\section{MODELING}

In order to further characterize the elongated structure detected with aperture synthesis imaging, we fitted geometric and detailed physical models to our data, allowing us to deduce object information on smaller scales than the diffraction-limited resolution of our reconstructed image.

\subsection{Geometric models}

Our measurements show that the visibility function drops rapidly at baseline lengths $\lesssim 3 \mathrm{~m}$, but stays then nearly constant up to baseline lengths of about $12 \mathrm{~m}$, followed by a uniform, nearly linear decline at baseline lengths up to $85 \mathrm{~m}$ (Fig. 1, right), providing clear evidence for the presence of at least two spatial components. We use a Gaussian model to estimate the characteristic size of the two components and find that the first, extended component has a FWHM diameter of about 108 mas and contributes $\sim 15 \%$ of the total flux. Based on the finding of scattered light envelopes around other massive stars, we suggest that this extended, centro-symmetric component, which also appears in our image as homogenously distributed flux elements, is the scattered light contribution of a natal envelope. The second, compact component accounts for $85 \%$ of the total $K$-band flux and has a size of $5.4 \times 3.8$ mas, consistent with a disk seen under an inclination angle of $45^{\circ}$. Furthermore, we find that the size of the compact component increases significantly towards longer wavelengths, as shown by the measured equivalent widths in Fig. $3(t o p)$. This effect, which was found towards many low- and intermediatemass YSO disks 24, 25 , is likely indicating a temperature gradient in the circumstellar material, where hotter material (radiating more effectively at shorter wavelengths) is located closer to the star.

Motivated by these indications for an internal temperature structure, we fitted analytical disk models with a radial temperature power-law, $T(r)=T_{\text {in }}\left(r / r_{\text {in }}\right)^{-q}$, to our data. Assuming reasonable values for the inner disk temperature, i.e. $T_{\mathrm{in}}=1500$ to $2000 \mathrm{~K}$, we find that this model can reproduce our interferometric data with an inner disk radius $r_{\text {in }}$ of 3 mas $(9.5 \mathrm{AU})$, and a temperature power law index $q$ of 0.4 , which is consistent with the theoretical temperature gradient of flared irradiated disks ${ }^{26}(q=0.43)$. Compared to other geometric models (e.g. uniform disk model with $\chi^{2}=4.48$ or Gaussian model with $\chi^{2}=2.56$ ), the temperature gradient disk model provides a significantly better representation of our data $\left(\chi^{2}=1.40\right)$. Intriguingly, the derived inner disk radius of 9.5 AU agrees with the expected location where dust in an irradiated circumstellar disk would sublimate, i.e. 6.2 AU to 10.9 AU assuming grey dust and dust sublimation temperatures between 2000 and $1500 \mathrm{~K}$. Therefore, IRAS 13481-6124 is following the size-luminosity relation 27 , which is well established for low- to intermediate mass YSOs, suggesting that the near-infrared emission mainly traces material at the dust sublimation radius, similar as in the disks around T Tauri and Herbig Ae/Be stars.

The derived disk orientation $\left(120^{\circ}\right.$, Fig. 20 D) is perpendicular to the determined outflow axis $\left(26 \pm 9^{\circ}\right.$ and $\left.31 \pm 6^{\circ}\right)$, suggesting that the AU-scale disk resolved by VLTI/AMBER is indeed the driving engine of the detected collimated outflow.

\subsection{Radiative transfer modeling}

In order to confirm that the proposed scenario, including a high-mass young stellar object, a compact disk, and an extended circumstellar envelope, can fit not only our interferometric observations but is also consistent with the measured SED, we performed detailed radiative transfer simulations. To obtain an initial parameter set for our modeling procedure, we used the pre-computed grid of 2-D radiative transfer models by Robitaille et 

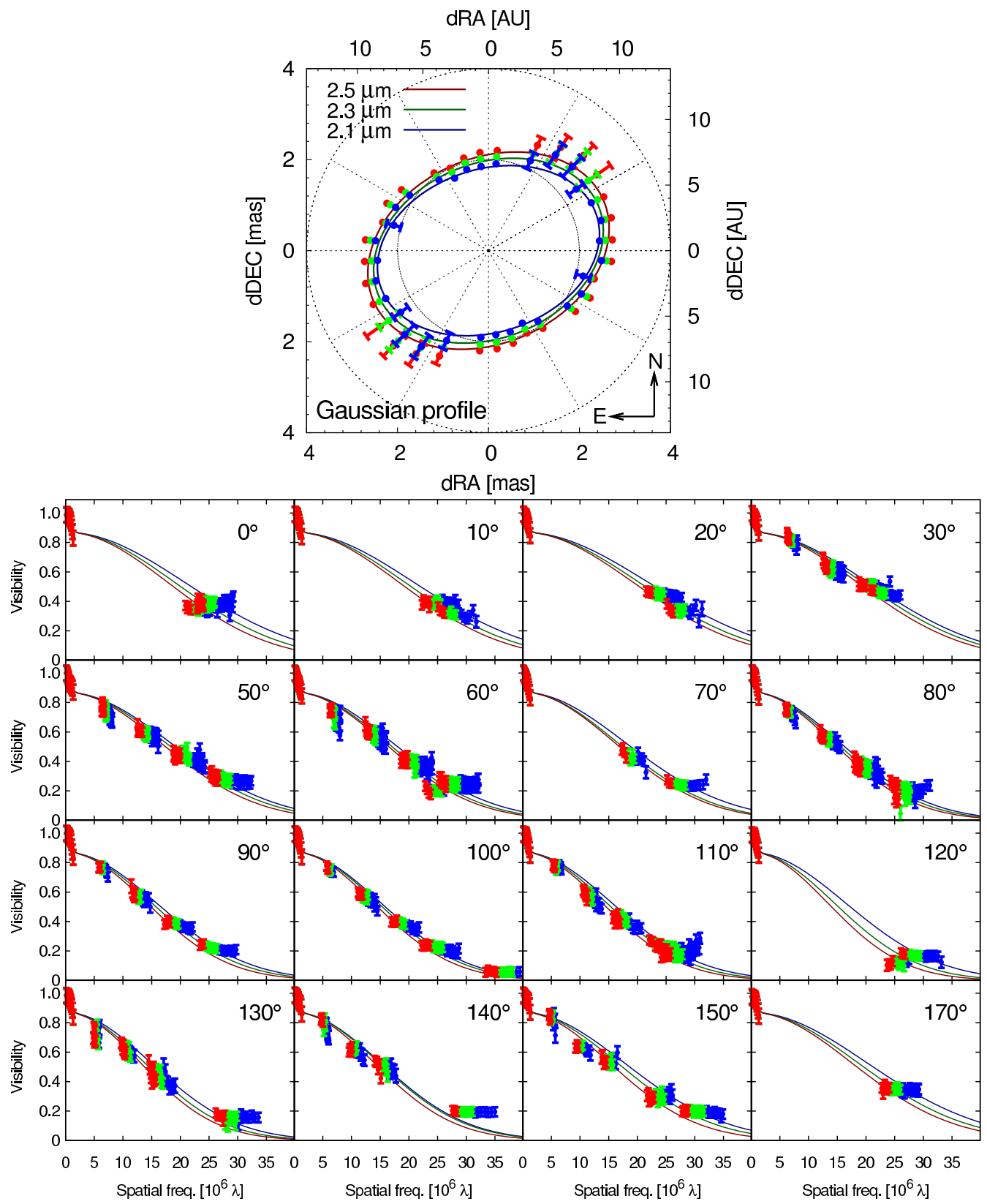

Figure 3. Elongation of the near-infrared emitting region (top), as determined by fitting Gaussian models to independent subsets of our visibility data (bottom), each covering a position angle range of $10^{\circ}$. In order to investigate whether the object morphology changes with wavelength, we have divided our data in three wavelength bins (blue: $\lambda<2.2$; green: $2.2 \leq \lambda<2.4$; red: $\lambda \geq 2.4$ ) and then adjusted the Gaussian FHWM diameter. Besides the compact component, this model also includes a Gaussian with FWHM diameter 108 mas, representing the extended envelope component detected with our NTT/speckle observations. 

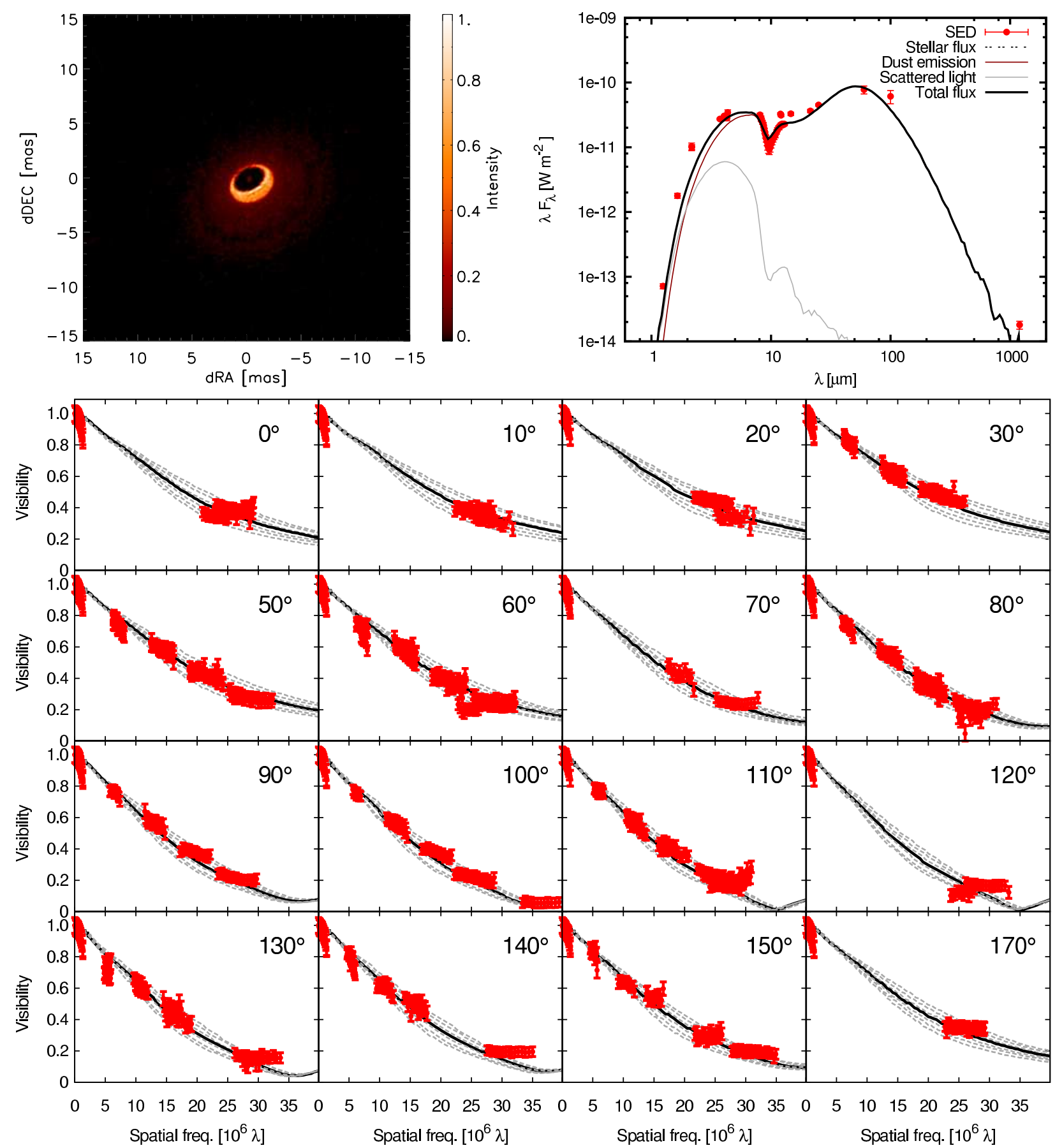

Figure 4. Top: Model image (left, $\lambda=2.25 \mu \mathrm{m}$ ) and SED (right) corresponding to our 2-D radiative transfer model. Bottom: Comparison of the measured and the model visibilities for the wavelength range covered by our AMBER observations (1.95 to $2.55 \mu \mathrm{m}$, grey dashed lines). The black lines show the visibility function for the central wavelength of $2.25 \mu \mathrm{m}$. 
al. $\stackrel{28}{28}$. The model parameters are then put into an adapted version of the radiative code by Whitney et al. 29 in order to compute model SEDs and synthetic images, from which we compute visibilities and closure phases for comparison with our interferometric observations. After some parameter adjustments we find a parameter set which reproduces the SED and the measured visibilities and closure phases reasonably well (Fig. 4). The model includes a central star with a mass of $\sim 18 \mathrm{M}_{\odot}$, a circumstellar disk with a curved rim and an inner dust truncation radius at $6.2 \mathrm{AU}$, and an extended envelope with bipolar outflow cavities. For a detailed description of the model components and the best-fit parameters, we refer the reader to our recent science paper $\underline{9}$.

\section{CONCLUSIONS}

Probing the dust distribution around a young high-mass star on the smallest physical scales yet, our study provides compelling evidence for the accretion-disk hypothesis in high-mass star formation and permits a first characterization of the geometry of the innermost disk regions. Furthermore, our study demonstrates the benefits of a multi-wavelength $(2-870 \mu \mathrm{m})$ and multi-scale imaging approach, which allows us to relate the detected AU-size compact disk with the outflow signatures detected on parsec scales, yielding a global picture of the environment around this massive young star.

With the available near-infrared interferometric instrumentation, the number of accessible high-mass YSO targets is rather limited, in particular due to the current sensitivity limits and the need for off-axis telescope guiding. However, the number of accessible targets might increase considerably with the upcoming generation of infrared interferometric instruments. For instance, the implementation of infrared wavefront sensing units would enable interferometric observations on a large number of targets without suitable visual guide stars. For VLTI, this implementation of near-infrared wavefront sensors $(H$ - or $K$-band) is planned in the course of the GRAVITY project ${ }^{30}$.

Besides these improvements for telescope guiding and beam injection, further progress could be achieved by observing in an optimal wavelength regime. Near-infrared ( $H$ - and $K$-band) interferometric observations provide an excellent angular resolution and already enable phase-closure imaging observations, but are restricted to a small sample of targets. In the mid-infrared $N$-band $(8-13 \mu \mathrm{m})$, the brightness limitations are significantly relaxed, while at these wavelengths the disk contributions get more contaminated with emission from the envelope and the angular resolution becomes insufficient to study the inner disk truncation region. Therefore, it is likely that the optimal observing wavelength for many young high-mass stars is between the $K$ - and $N$-band, for instance in the $L$ - or $M$-band. Two-telescope $L$-band interferometry was recently demonstrated at the Keck Interferometer ${ }^{31}$ and will also become available with the VLTI second-generation instrument MATISSE 32 , enabling four-telescope $L$ - or $M$-band interferometric imaging on a large sample of massive YSOs.

\section{ACKNOWLEDGMENTS}

This work was performed in part under contract with the California Institute of Technology (Caltech) funded by NASA through the Sagan Fellowship Program. We would like to thank the ESO Paranal staff for their support during our observing runs and their enduring efforts in improving VLTI. This paper is based on observations made with ESO telescopes at the La Silla Paranal Observatory and used archival data obtained with the Spitzer Space Telescope, which is operated by the Jet Propulsion Laboratory, California Institute of Technology under a contract with NASA, and on data acquired with the Atacama Pathfinder Experiment (APEX). APEX is a collaboration between the Max-Planck-Institut für Radioastronomie, ESO, and the Onsala Space Observatory. 


\section{REFERENCES}

1. Kahn, F. D., "Cocoons around early-type stars," A\&A 37, 149-162 (Dec. 1974).

2. Wolfire, M. G. and Cassinelli, J. P., "Conditions for the formation of massive stars," ApJ 319, 850-867 (Aug. 1987).

3. Bonnell, I. A., Bate, M. R., and Zinnecker, H., "On the formation of massive stars," MNRAS 298, 93-102 (July 1998).

4. McKee, C. F. and Tan, J. C., "Massive star formation in 100,000 years from turbulent and pressurized molecular clouds," Nature 416, 59-61 (Mar. 2002).

5. Krumholz, M. R., Klein, R. I., McKee, C. F., Offner, S. S. R., and Cunningham, A. J., "The Formation of Massive Star Systems by Accretion," Science 323, 754- (Feb. 2009).

6. Beltrán, M. T., Cesaroni, R., Codella, C., Testi, L., Furuya, R. S., and Olmi, L., "Infall of gas as the formation mechanism of stars up to 20 times more massive than the Sun," Nature 443, 427-429 (Sept. 2006).

7. Linz, H., Henning, T., Feldt, M., Pascucci, I., van Boekel, R., Men'shchikov, A., Stecklum, B., Chesneau, O., Ratzka, T., Quanz, S. P., Leinert, C., Waters, L. B. F. M., and Zinnecker, H., "Mid-infrared interferometry of massive young stellar objects. I. VLTI and Subaru observations of the enigmatic object M8E-IR," $A \mathscr{E} A$ 505, 655-661 (Oct. 2009).

8. de Wit, W. J., Hoare, M. G., Oudmaijer, R. D., and Lumsden, S. L., "The origin of mid-infrared emission in massive young stellar objects: multi-baseline VLTI observations of W33A," ArXiv e-prints (Dec. 2009).

9. Kraus, S., Hofmann, K.-H., Menten, K., Schertl, D., Weigelt, G., Wyrowski, F., Meilland, A., Perraut, K., Petrov, R., Robbe-Dubois, S., Schilke, P., and Testi, L., "A hot compact dust disk around a massive young stellar object," Nature, in press (June 2010).

10. Fontani, F., Beltrán, M. T., Brand, J., Cesaroni, R., Testi, L., Molinari, S., and Walmsley, C. M., "Search for massive protostellar candidates in the southern hemisphere. I. Association with dense gas," $A \& A$ 432, 921-935 (Mar. 2005).

11. Grave, J. M. C. and Kumar, M. S. N., "Spitzer-IRAC GLIMPSE of high mass protostellar objects. II. SED modelling of a bona fide sample," A\&A 498, 147-159 (Apr. 2009).

12. Beltrán, M. T., Brand, J., Cesaroni, R., Fontani, F., Pezzuto, S., Testi, L., and Molinari, S., "Search for massive protostar candidates in the southern hemisphere. II. Dust continuum emission," A $\& A$ 447, 221-233 (Feb. 2006).

13. Petrov, R. G., Malbet, F., Weigelt, G., Antonelli, P., Beckmann, U., Bresson, Y., Chelli, A., Dugué, M., Duvert, G., Gennari, S., Glück, L., Kern, P., Lagarde, S., Le Coarer, E., Lisi, F., Millour, F., Perraut, K., Puget, P., Rantakyrö, F., Robbe-Dubois, S., Roussel, A., Salinari, P., Tatulli, E., Zins, G., Accardo, M., Acke, B., Agabi, K., Altariba, E., Arezki, B., Aristidi, E., Baffa, C., Behrend, J., Blöcker, T., Bonhomme, S., Busoni, S., Cassaing, F., Clausse, J.-M., Colin, J., Connot, C., Delboulbé, A., Domiciano de Souza, A., Driebe, T., Feautrier, P., Ferruzzi, D., Forveille, T., Fossat, E., Foy, R., Fraix-Burnet, D., Gallardo, A., Giani, E., Gil, C., Glentzlin, A., Heiden, M., Heininger, M., Hernandez Utrera, O., Hofmann, K.-H., Kamm, D., Kiekebusch, M., Kraus, S., Le Contel, D., Le Contel, J.-M., Lesourd, T., Lopez, B., Lopez, M., Magnard, Y., Marconi, A., Mars, G., Martinot-Lagarde, G., Mathias, P., Mège, P., Monin, J.-L., Mouillet, D., Mourard, D., Nussbaum, E., Ohnaka, K., Pacheco, J., Perrier, C., Rabbia, Y., Rebattu, S., Reynaud, F., Richichi, A., Robini, A., Sacchettini, M., Schertl, D., Schöller, M., Solscheid, W., Spang, A., Stee, P., Stefanini, P., Tallon, M., Tallon-Bosc, I., Tasso, D., Testi, L., Vakili, F., von der Lühe, O., Valtier, J.-C., Vannier, M., and Ventura, N., "AMBER, the near-infrared spectro-interferometric three-telescope VLTI instrument," $A \mathscr{E} A$ 464, 1-12 (Mar. 2007).

14. Labeyrie, A., "Attainment of Diffraction Limited Resolution in Large Telescopes by Fourier Analysing Speckle Patterns in Star Images," A\&A 6, 85-+ (May 1970).

15. Weigelt, G. P., "Modified astronomical speckle interferometry 'speckle masking'," Optics Communications 21, 55-59 (Apr. 1977).

16. Benjamin, R. A., Churchwell, E., Babler, B. L., Bania, T. M., Clemens, D. P., Cohen, M., Dickey, J. M., Indebetouw, R., Jackson, J. M., Kobulnicky, H. A., Lazarian, A., Marston, A. P., Mathis, J. S., Meade, M. R., Seager, S., Stolovy, S. R., Watson, C., Whitney, B. A., Wolff, M. J., and Wolfire, M. G., "GLIMPSE. I. An SIRTF Legacy Project to Map the Inner Galaxy," PASP 115, 953-964 (Aug. 2003). 
17. Smith, H. A., Hora, J. L., Marengo, M., and Pipher, J. L., "Outflows from Massive Young Stellar Objects as Seen with the Infrared Array Camera," ApJ 645, 1264-1271 (July 2006).

18. Hofmann, K.-H. and Weigelt, G., "Iterative image reconstruction from the bispectrum," $A \& A$ 278, 328-339 (Oct. 1993).

19. Lawson, P. R., Cotton, W. D., Hummel, C. A., Baron, F., Young, J. S., Kraus, S., Hofmann, K., Weigelt, G. P., Ireland, M., Monnier, J. D., Thiébaut, E., Rengaswamy, S., and Chesneau, O., "2006 interferometry imaging beauty contest," in [Society of Photo-Optical Instrumentation Engineers (SPIE) Conference Series], 6268 (July 2006).

20. Cotton, W., Monnier, J., Baron, F., Hofmann, K., Kraus, S., Weigelt, G., Rengaswamy, S., Thiébaut, E., Lawson, P., Jaffe, W., Hummel, C., Pauls, T., Schmitt, H., Tuthill, P., and Young, J., "2008 imaging beauty contest," in [Society of Photo-Optical Instrumentation Engineers (SPIE) Conference Series], 7013 (July 2008).

21. Kraus, S., Balega, Y. Y., Berger, J., Hofmann, K., Millan-Gabet, R., Monnier, J. D., Ohnaka, K., Pedretti, E., Preibisch, T., Schertl, D., Schloerb, F. P., Traub, W. A., and Weigelt, G., "Visual/infrared interferometry of Orion Trapezium stars: preliminary dynamical orbit and aperture synthesis imaging of the $\theta^{1}$ Orionis $\mathrm{C}$ system," A\&A 466, 649-659 (May 2007).

22. Kraus, S., Weigelt, G., Balega, Y. Y., Docobo, J. A., Hofmann, K.-H., Preibisch, T., Schertl, D., Tamazian, V. S., Driebe, T., Ohnaka, K., Petrov, R., Schöller, M., and Smith, M., "Tracing the young massive higheccentricity binary system $\theta^{1}$ Orionis C through periastron passage," A\& $A 497,195-207$ (Apr. 2009).

23. Millour, F., Chesneau, O., Borges Fernandes, M., Meilland, A., Mars, G., Benoist, C., Thiébaut, E., Stee, P., Hofmann, K., Baron, F., Young, J., Bendjoya, P., Carciofi, A., Domiciano de Souza, A., Driebe, T., Jankov, S., Kervella, P., Petrov, R. G., Robbe-Dubois, S., Vakili, F., Waters, L. B. F. M., and Weigelt, G., "A binary engine fuelling HD 87643's complex circumstellar environment. Determined using AMBER/VLTI imaging," $A \mathscr{E} A$ 507, 317-326 (Nov. 2009).

24. Eisner, J. A., Chiang, E. I., Lane, B. F., and Akeson, R. L., "Spectrally Dispersed K-Band Interferometric Observations of Herbig Ae/Be Sources: Inner Disk Temperature Profiles," ApJ 657, 347-358 (Mar. 2007).

25. Kraus, S., Preibisch, T., and Ohnaka, K., "Detection of an Inner Gaseous Component in a Herbig Be Star Accretion Disk: Near- and Mid-Infrared Spectrointerferometry and Radiative Transfer modeling of MWC 147," ApJ 676, 490-508 (Mar. 2008).

26. Chiang, E. I. and Goldreich, P., "Spectral Energy Distributions of T Tauri Stars with Passive Circumstellar Disks," ApJ 490, 368-+ (Nov. 1997).

27. Monnier, J. D., Millan-Gabet, R., Billmeier, R., Akeson, R. L., Wallace, D., Berger, J.-P., Calvet, N., D’Alessio, P., Danchi, W. C., Hartmann, L., Hillenbrand, L. A., Kuchner, M., Rajagopal, J., Traub, W. A., Tuthill, P. G., Boden, A., Booth, A., Colavita, M., Gathright, J., Hrynevych, M., Le Mignant, D., Ligon, R., Neyman, C., Swain, M., Thompson, R., Vasisht, G., Wizinowich, P., Beichman, C., Beletic, J., CreechEakman, M., Koresko, C., Sargent, A., Shao, M., and van Belle, G., "The Near-Infrared Size-Luminosity Relations for Herbig Ae/Be Disks," ApJ 624, 832-840 (May 2005).

28. Robitaille, T. P., Whitney, B. A., Indebetouw, R., Wood, K., and Denzmore, P., "Interpreting Spectral Energy Distributions from Young Stellar Objects. I. A Grid of 200,000 YSO Model SEDs," ApJS 167, 256-285 (Dec. 2006).

29. Whitney, B. A., Wood, K., Bjorkman, J. E., and Wolff, M. J., "Two-dimensional Radiative Transfer in Protostellar Envelopes. I. Effects of Geometry on Class I Sources," ApJ 591, 1049-1063 (July 2003).

30. Hippler, S., Brandner, W., Clénet, Y., Hormuth, F., Gendron, E., Henning, T., Klein, R., Lenzen, R., Meschke, D., Naranjo, V., Neumann, U., Ramos, J. R., Rohloff, R., and Eisenhauer, F., "Near-infrared wavefront sensing for the VLT interferometer," in [Society of Photo-Optical Instrumentation Engineers (SPIE) Conference Series], Presented at the Society of Photo-Optical Instrumentation Engineers (SPIE) Conference $\mathbf{7 0 1 5}$ (July 2008).

31. Ragland, S., Akeson, R. L., Armandroff, T., Colavita, M. M., Danchi, W. C., Hillenbrand, L. A., MillanGabet, R., Ridgway, S. T., Traub, W. A., Vasisht, G., and Wizinowich, P. L., "First L-Band Interferometric Observations of a Young Stellar Object: Probing the Circumstellar Environment of MWC 419," ApJ 703, 22-29 (Sept. 2009). 
32. Lopez, B., Antonelli, P., Wolf, S., Lagarde, S., Jaffe, W., Navarro, R., Graser, U., Petrov, R., Weigelt, G., Bresson, Y., Hofmann, K. H., Beckman, U., Henning, T., Laun, W., Leinert, C., Kraus, S., Robbe-Dubois, S., Vakili, F., Richichi, A., Abraham, P., Augereau, J., Behrend, J., Berio, P., Berruyer, N., Chesneau, O., Clausse, J. M., Connot, C., Demyk, K., Danchi, W. C., Dugué, M., Finger, G., Flament, S., Glazenborg, A., Hannenburg, H., Heininger, M., Hugues, Y., Hron, J., Jankov, S., Kerschbaum, F., Kroes, G., Linz, H., Lizon, J., Mathias, P., Mathar, R., Matter, A., Menut, J. L., Meisenheimer, K., Millour, F., Nardetto, N., Neumann, U., Nussbaum, E., Niedzielski, A., Mosoni, L., Olofsson, J., Rabbia, Y., Ratzka, T., Rigal, F., Roussel, A., Schertl, D., Schmider, F., Stecklum, B., Thiebaut, E., Vannier, M., Valat, B., Wagner, K., and Waters, L. B. F. M., "MATISSE: perspective of imaging in the mid-infrared at the VLTI," in [Society of Photo-Optical Instrumentation Engineers (SPIE) Conference Series], Society of Photo-Optical Instrumentation Engineers (SPIE) Conference Series $\mathbf{7 0 1 3}$ (July 2008). 


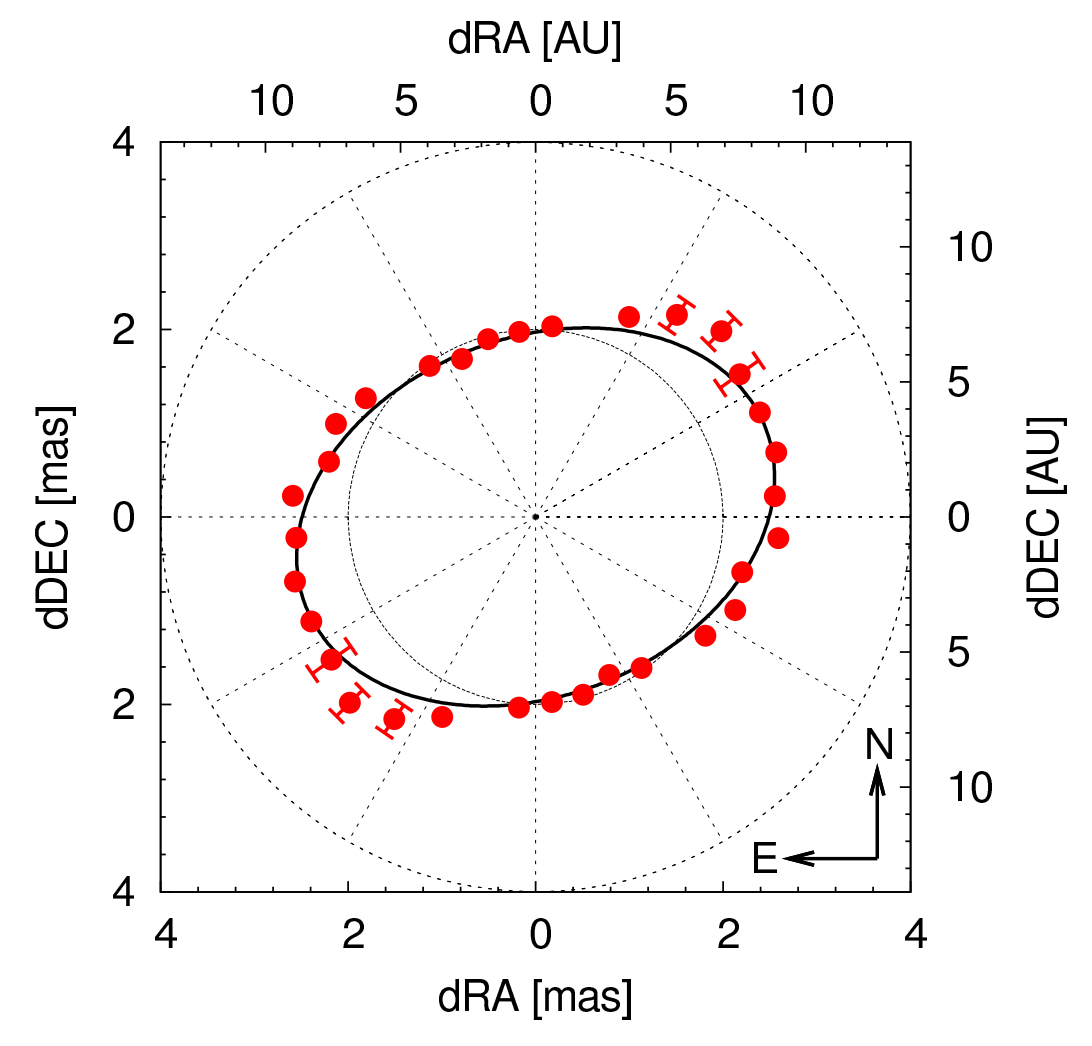

